\title{
Assessing Influence of Solution-focused Nursing for Severe Acute Pancreatitis Patients in Double Filtration Plasmapheresis
}

\author{
Yanyun Chen ${ }^{1}$, Zhaolin Chen ${ }^{2,}$, , Jieyu Rao ${ }^{1}$, Xiaoxi Cai ${ }^{1}$, Hongcheng Wei ${ }^{1}$ \\ ${ }^{1}$ Artificial Liver Blood Purification Department, The First Affiliated Hospital of Jinan University, Guangzhou, China \\ ${ }^{2}$ Gastroenterology Department, The First Affiliated Hospital of Jinan University, Guangzhou, China \\ Email address: \\ 499324016@qq.com (Yanyun Chen),2009gzjnu@163.com (Zhaolin Chen), 546888129@qq.com (Jieyu Rao), \\ 2206997396@qq.com (Xiaoxi Cai), twhch@jnu.edu.cn (Hongcheng Wei) \\ ${ }^{*}$ Corresponding author
}

\section{To cite this article:}

Yanyun Chen, Zhaolin Chen, Jieyu Rao, Xiaoxi Cai, Hongcheng Wei. Assessing Influence of Solution-focused Nursing for Severe Acute Pancreatitis Patients in Double Filtration Plasmapheresis. Science Journal of Clinical Medicine. Vol. 9, No. 1, 2020, pp. 11-14.

doi: $10.11648 /$ j.sjcm.20200901.14

Received: March 1, 2020; Accepted: March 13, 2020; Published: March 23, 2020

\begin{abstract}
Objective: To evaluate influence of solution-focused nursing for severe acute pancreatitis patients in Double Filtration Plasmapheresis. Methods: 260 patients were invited join our study who were diagnosed as acute pancreatitis, they undergoing Double Filtration Plasmapheresis from January 2015 to September 2019. The participants assigned randomly into the intervention group and control group. The control group patient receive traditional nursing services in treatment process. Also, the intervention group patient receive solution-focused nursing services. Additionally, we collected the data from all participants, the data included anxiety information, depression information, self-care ability, self-management effectiveness, Serum amylase, Urine amylase, tumor necrosis factor- $\alpha$ and interleukin- 6. The used questionnaires include Self-Rating Anxiety Scale (SAS), Self-rating depression scale (SDS), exercise of self-care a- gency (ESCA) and Chronic Disease Self Efficacy Scales Stanfor (SESC). Result: The intervention group had greater score in SDS and SAS, but the gap of improvement between intervention group and control group was not big. In 4 domains of ESCA, all improvements were undistinguished in control group. Besides, the self-management ability improvement of two groups was slightly in the result, but intervention group had greater performance than that of control group in all domains. Conclusion: The solution focused nursing improve the outcome of DFPP treatment with AP patient in Chinese hospital. The improvement included 4 domains in the result, such as mental health (anxiety $\&$ depression), self-care ability, self-management ability and recovery status. But the simple size was limit the accuracy of results.
\end{abstract}

Keywords: Solution-focused Nursing, Acute Pancreatitis, Double Filtration Plasmapheresis

\section{Introduction}

Double Filtration Plasmapheresis (DFPP) is a general treatment method with patients with liver failure, has been used increasingly for induction of immunotolerance in high-risk operation [1-3]. In some Chinese hospital, DFPP teams have been assembled to manage perioperative DFPP. This approach allows for improved efficiency and reduced time to therapy $[4,5]$. In addition, the DFPP team typically consists 6 roles, such as transfusion medicine physician, doctor with severe acute pancreatitis, perfusionist, pharmacist, anesthesiologist and critical care nurses [6]. Base on report, DFPP's therapeutic effect is secondary to elimination of antibodies, immune complexes, and cytokines in plasma with the premise that removal of these mediators will ameliorate disease [7, 8]. DFPP is an apheresis procedure where the patient's plasma is separated, removed, and simultaneously replaced with another fluid to maintain a normal blood volume [9]. In 1914, the remaining blood fraction was re-introduced into the patient, patient's blood was removed from patients, plasma was discarded [10]. In addition, acute pancreatitis (AP) is one of the most common gastrointestinal disorders with 
morbidity of 13-45/100,00 worldwide [11]. Approximately $10 \% \sim 20 \%$ of AP cases will progress to severe acute pancreatitis (SAP), which is associated with rapid progression, multiple complications and high mortalit [12].

Solution focused nursing (SFN) is a model of care that offers an alternative approach to engaging with clients, and to the discipline of nursing itself, an approach based on respect for the individual, and faith in that person's own resources and potential $[13,14]$. Basically the difference between solution focused nursing and traditional nursing is an alternative way of thinking about problems and issues. There is increasing evidence that solution focused models of care do offer potential for improved outcomes [15, 16]. Base on some reports of of all available outcomes studies on solution focused care available at time of reporting, five were well controlled, and four of these showed the solution focus to be better than standard care $[13,17]$. The aim of our study is assess influence of solution-focused nursing for severe acute pancreatitis patients in double filtration Double Filtration Plasmapheresis.

\section{Methods}

\subsection{Participants Enrollment and Survey Methods}

260 patients who were diagnosed as acute pancreatitis were investigated join into our study, all of them undergoing Double Filtration Plasmapheresis from January 2015 to September 2019. We randomly assigned the participants to the control group $(n=130)$ and the intervention group $(n=130)$. In different groups, the patients were receive different nursing measure in treatment process. For one things, the patients of control group had traditional nursing services from our researchers. For another things, the patients of intervention group were receive solution focused nursing. We collected the data from all participants, the data included anxiety information, depression information, self-care ability, self-management effectiveness, Serum amylase, Urine amylase, tumor necrosis factor- $\alpha$ (TNF- $\alpha)$ and interleukin- 6 (IL-6). We collected data using the following questionnaires; Self-Rating Anxiety Scale (SAS), Self-rating depression scale (SDS), exercise of self-care a- gency (ESCA) and Chronic Disease Self Efficacy Scales Stanfor (SESC). Another data was from patient record and assay result $[18,19]$.

Their inclusion criteria were: (1) the patients were diagnosed as acute pancreatitis; (2) They undergoing Double Filtration Plasmapheresis; (3) Patients volunteered to participate in follow-up; Their withdraw criteria were: (1) the patients had too many complications; (2) They had other pancreatic problems.

\subsection{Statistical Analysis}

Survey responses were analyzed using descriptive statistics. Sample proportions, means, and standard deviation (SD) are reported. All analyses were performed in SPSS 24.

\section{Result}

In the result of SAS and SDS, the intervention group had greater score, but the gap of improvement between intervention group and control group was not big (Table 1). For depression status of patient, the effect of traditional nursing service was not significant in the outcome (from $71.03 \pm 7.21$ to $55.13 \pm 6.27$ vs from $71.14 \pm 7.54$ to $62.54 \pm 6.35$ ).

Table 1. The outcome of SAS and SDS (Mean $\pm S D$ ).

\begin{tabular}{|c|c|c|c|c|c|c|c|c|}
\hline \multirow{2}{*}{ Projects } & \multicolumn{2}{|l|}{ SAS } & \multirow{2}{*}{$\mathbf{T}$} & \multirow{2}{*}{ P value } & \multicolumn{2}{|l|}{ SDS } & \multirow{2}{*}{$\mathbf{T}$} & \multirow{2}{*}{$P$ value } \\
\hline & BI & FI & & & BI & FI & & \\
\hline Intervention Group $(\mathrm{n}=130)$ & $69.36 \pm 7.51$ & $52.34 \pm 5.24$ & 23.1237 & $<0.005$ & $71.03 \pm 7.21$ & $55.13 \pm 6.27$ & 32.9367 & $<0.005$ \\
\hline Control Group $(n=130)$ & $70.02 \pm 7.71$ & $58.34 \pm 5.31$ & 12.5822 & $<0.005$ & $71.14 \pm 7.54$ & $62.54 \pm 6.35$ & 17.3510 & $<0.005$ \\
\hline $\mathrm{T}$ & 1.312 & 27.251 & - & - & 0.0172 & 13.5902 & - & - \\
\hline$P$ value & 0.594 & 0.021 & - & - & 0.381 & 0.149 & - & - \\
\hline
\end{tabular}

SAS $=$ Self-Rating Anxiety Scale

SDS $=$ Self-rating depression scale

$\mathrm{BI}=$ before the intervention

$\mathrm{FI}=$ after the intervention

In the total of self-care ability, the patients of intervention group had stronger self-care ability after the intervention than that of control group $(125.36 \pm 16.54$ vs $107.82 \pm 15.69)$ (Table 2). In 4 domains of ESCA, all improvements were undistinguished in control group. The different is that the patient of intervention group received greatest improvement in Self-care knowledge (from $21.26 \pm 4.28$ to $34.47 \pm 5.21$ ).

Table 2. Self-care ability (Mean $\pm S D$ ).

\begin{tabular}{|c|c|c|c|c|c|c|c|}
\hline Projects & Period & Self concept & $\begin{array}{l}\text { Self-care } \\
\text { responsibility }\end{array}$ & $\begin{array}{l}\text { Self-care } \\
\text { knowledge }\end{array}$ & Self-care skills & Total & P Value \\
\hline \multirow{2}{*}{ Intervention Group $(\mathrm{n}=130)$} & $\mathrm{BI}$ & $23.34 \pm 3.58$ & $24.21 \pm 5.11$ & $21.26 \pm 4.28$ & $24.33 \pm 5.14$ & $93.14 \pm 12.31$ & $<0.005$ \\
\hline & FI & $31.21 \pm 4.58$ & $30.36 \pm 5.16$ & $34.47 \pm 5.21$ & $29.31 \pm 6.11$ & $125.36 \pm 16.54$ & 0.134 \\
\hline \multirow{2}{*}{ Control Group $(n=130)$} & $\mathrm{BI}$ & $23.44 \pm 3.77$ & $24.31 \pm 5.37$ & $21.22 \pm 4.11$ & $24.57 \pm 5.16$ & $93.54 \pm 12.22$ & 0.042 \\
\hline & FI & $26.35 \pm 4.62$ & $27.23 \pm 4.89$ & $27.66 \pm 5.13$ & $26.58 \pm 6.27$ & $107.82 \pm 15.69$ & $<0.005$ \\
\hline
\end{tabular}

$\mathrm{BI}=$ before the intervention

$\mathrm{FI}=$ after the intervention 
Base on Table 3, it shown the self-management of patient at before intervention and after intervention. Overall, the improvement of two groups was slightly in the result, but intervention group had greater performance than that of control group in all domains. In particular, compared with the other domains, the emotion management score of intervention group had biggest change, the emotion management score of intervention group was from $4.68 \pm 0.69$ to $8.13 \pm 2.14$.

Table 3. Self-management effectiveness (Mean $\pm S D)$.

\begin{tabular}{|c|c|c|c|c|c|c|c|}
\hline Projects & Period & $\begin{array}{l}\text { Symptom } \\
\text { management }\end{array}$ & $\begin{array}{l}\text { Emotion } \\
\text { management }\end{array}$ & $\begin{array}{l}\text { Self } \\
\text { management }\end{array}$ & $\begin{array}{l}\text { Fatigue } \\
\text { management }\end{array}$ & $\begin{array}{l}\text { Drug } \\
\text { administration }\end{array}$ & $\begin{array}{l}\text { Pain } \\
\text { management }\end{array}$ \\
\hline \multirow{2}{*}{ Intervention Group $(\mathrm{n}=130)$} & $\mathrm{BI}$ & $5.11 \pm 1.05$ & $4.68 \pm 0.69$ & $5.39 \pm 1.24$ & $4.69 \pm 1.17$ & $6.02 \pm 1.43$ & $5.02 \pm 1.08$ \\
\hline & FI & $7.75 \pm 1.35$ & $8.13 \pm 2.14$ & $7.88 \pm 1.68$ & $6.98 \pm 1.46$ & $8.79 \pm 2.21$ & $7.75 \pm 1.16$ \\
\hline \multirow{2}{*}{ Control Group $(n=130)$} & BI & $5.13 \pm 1.12$ & $4.71 \pm 0.73$ & $5.41 \pm 1.26$ & $4.72 \pm 4.21$ & $6.11 \pm 1.44$ & $5.06 \pm 1.13$ \\
\hline & FI & $6.57 \pm 1.36$ & $6.21 \pm 2.11$ & $6.24 \pm 1.72$ & $5.44 \pm 1.42$ & $7.16 \pm 1.86$ & $6.27 \pm 1.05$ \\
\hline
\end{tabular}

$\mathrm{BI}=$ before the intervention

$\mathrm{FI}=$ after the intervention

The data of serum biochemical index and amylase index was from patient test in treatment process, it included 4 domains, such as TNF- $\alpha$, IL-6, Serum amylase and Urine amylase (Table 2). Those data shown the change of acute pancreatitis status of patient. Base on Table 2, the intervention group had better recovery in the result that that of control group, but the improvement was not significant in the serum biochemical index and amylase index.

Table 4. Serum amylase, Urine amylase, tumor necrosis factor- $\alpha$ (TNF- $\alpha$ ) and interleukin- 6 (IL-6) (Mean $\pm S D)$.

\begin{tabular}{|c|c|c|c|c|c|}
\hline \multirow{2}{*}{ Projects } & \multirow{2}{*}{ Period } & \multicolumn{2}{|c|}{ Serum biochemical index } & \multicolumn{2}{|l|}{ Amylase Index } \\
\hline & & TNF- $\alpha(n g / L)$ & IL-6 (ng/L) & Serum amylase (U/L) & Urine amylase (U/L) \\
\hline \multirow{2}{*}{ Intervention Group $(\mathrm{n}=130)$} & $\mathrm{BI}$ & $395.27 \pm 35.26$ & $78.98 \pm 12.58$ & $171.13 \pm 26.21$ & $1555.13 \pm 336.77$ \\
\hline & FI & $256.69 \pm 26.58$ & $53.24 \pm 10.74$ & $91.55 \pm 23.12$ & $709.65 \pm 332.16$ \\
\hline \multirow{2}{*}{ Control Group $(\mathrm{n}=130)$} & $\mathrm{BI}$ & $396.13 \pm 36.02$ & $78.71 \pm 12.41$ & $178.14 \pm 27.54$ & $1572.51 \pm 314.97$ \\
\hline & FI & $271.25 \pm 25.58$ & $59.71 \pm 11.71$ & $108.67 \pm 28.90$ & $1067.91 \pm 301.06$ \\
\hline $\mathrm{T}$ & - & 2.518 & 42.241 & 0.314 & 25.390 \\
\hline
\end{tabular}

$\mathrm{BI}=$ before the intervention

$\mathrm{FI}=$ after the intervention

\section{Discussion}

$\mathrm{AP}$ is the leading cause of gastroenterological hospitalisations. In the UK, the hospitalisation rate for acute pancreatitis is 9.8 per 100,000 per year, and is associated itself with significant morbidity and mortality [20]. Base on the reports, the AP patients have $10 \%$ progressing to chronic pancreatitis, because patients with acute pancreatitis have an increased risk of further reoccurrence [21]. Additionally, AP may lead to some complications, they may cause significant morbidity and even mortality. In fact, AP may result in transient (lasting $48 \mathrm{~h}$ ) or persistent $(>48 \mathrm{~h}$ ) organ failure and various local complications, including acute peri-pancreatic fluid collection, pancreatic necrosis, pseudocyst, and walled-off necrosis with or without infection.

Base on the result of this study, the solution focused nursing improve the outcome of DFPP treatment with AP patient in China. The improvement included 4 domains in the result, such as mental health (anxiety \& depression), self-care ability, self-management ability and recovery status. In mental health domain, the patients of intervention group had greater improvement in total score of SDS and SAS. Moreover, depression of intervention group patient was weaker than that of control group, that SDS result of intervention group patient had greater gap between before intervention and after intervention. Similarly, in self-care ability and self-management ability of patient, they had slight improvement in the treatment, it shown the solution focused nursing service provide medical image and service quality to the patient in the treatment process. However, parts of result were not statistical significance as simple size was limit the accuracy of results. The table 4 shown the AP status of patient in two groups, The AP status of total patient were sustained recovery, but the intervention group and control group had different recovery efficiency. Base on the result of test, the solution focused nursing had better recovery efficiency in the treatment process as its related index is closer to normal range.

\section{References}

[1] Tinckam KJ, Keshavjee S, Chaparro C, et al. Survival in sensitized lung transplant recipients with perioperative desensitization. Am J Transplant 2015; 15: 417-26.

[2] Djamali A, Kaufman DB, Ellis TM, Zhong W, Matas A, Samaniego M. Diagnosis and management of antibody-mediated rejection: current status and novel approaches. Am J Transplant 2014; 14: 255-71.

[3] Clark WF, Huang S-HS, Walsh MW, Farah M, Hildebrand AM, Sontrop JM. Plasma exchange for the treatment of kidney diseases. Kidney Int. 2016; 90 (5): 974-84. 
[4] Orandi BJ, Luo X, Massie AB, Garonzik-Wang JM, Lonze BE, Ahmed R, et al. Survival benefit with kidney transplants from HLA-Incompatible live donors. N Engl J Med. 2016; 374 (10): 940-950.

[5] Maillard N, Absi L, Claisse G, Masson I, Alamartine E, Mariat C. Protein A-Based immunoadsorption is more efficient than conventional plasma exchange to remove circulating Anti-HLA antibodies. Blood Purif. 2015; 40 (2): 167-172.

[6] Bridoux F, Carron P-L, Pegourie B, Alamartine E, Augeul-Meunier K, Karras A, et al. Effect of high-cutoff hemodialysis vs conventional hemodialysis on hemodialysis independence among patients with myeloma cast nephropathy: a randomized clinical trial. JAMA 2017; 318 (21): 2099-2110.

[7] Koizumi K, Hoshiai M, Moriguchi T, et al. Plasma Exchange Downregulates Activated Monocytes and Restores Regulatory $\mathrm{T}$ Cells in Kawasaki Disease. Therapeutic apheresis and dialysis: official peer-reviewed journal of the International Society for Apheresis, the Japanese Society for Apheresis, the Japanese Society for Dialysis Therapy. 2019; 23: 92-8.

[8] Yeh JH, Chien PJ, Hsueh YM, Shih CM, Chiu HC. Changes in the lymphocyte subset after double-filtration plasmapheresis. American journal of clinical pathology 2017; 128: 940-944.

[9] Bruel A, Kavanagh D, Noris M, Delmas Y, Wong EKS, Bresin $\mathrm{E}$, et al. Hemolytic uremic syndrome in pregnancy and postpartum. Clin J Am Soc Nephrol CJASN 2017; 12 (8): $1237-1247$.

[10] Abel JJ, Rowntree LG, Turner BB. Plasma removal with return of corpuscles (plasmaphaeresis). The Journal of Pharmacology and experimental therapeutics Vol. V. No. 6, July, 1914. Transfusion science. 1990; 11: 166-177.

[11] IAP/APA, evidence-based guidelines for the management of acute pancreatitis, Pancreatology. 2013; 13: e1-e15.
[12] Portelli M, Jones CD. Severe acute pancreatitis: pathogenesis, diagnosis and surgical management, Hepatob. Pancreat. Dis. 2017; 16: 155-159.

[13] McAllister M. Doing practice differently: solution focused nursing. J. Adv. Nurs. 2003; 41 (6): 528-535.

[14] Stevenson C, Jackson S, Barker P. Finding solutions through empowerment: A preliminary study of a solutionoriented approach to nursing in acute psychiatric settings. J. Psychiat. Mental Health Nurs. 2015; 10: 688-696.

[15] Barker P. The tidal model: the lived experience in person-centered mental health nursing care. Nurs. Philos. 2011 2 (3), 213-223.

[16] Bowles N, Mackintosh C, Torn A. Nurses' communication skills: An evaluation of the impact of solution-focused communication training. J. Adv. Nurs. 2017; 36 (3): 347-354.

[17] Vaughn K, Webster D, Orahood S, Young B. Brief inpatient psychiatric treatment: Finding solutions. Issues Mental Health Nurs. 2015; 16: 519-531.

[18] White D, Leach C, Sims R, Atkinson M, Cottrell D. Validation of the Hospital Anxiety and Depression Scale for use with adolescents. Br J Psychiatry 1999; 175: 452-454.

[19] El-Rufaie O, Absood G. Validity study of the Hospital Anxiety and Depression Scale among a group of Saudi patients. Br J Psychiatry 1987; 151: 687-688.

[20] Goldacre M. Hospital admission for acute pancreatitis in an English population, 1963-98: database study of incidence and mortality. BMJ. 2014; 328: 1466-1469.

[21] Yadav D, O'Connell M, Papachristou G. Natural history following the first attack of acute pancreatitis. Am. J. Gastroenterol. 2017; 107: 1096-1103. 